This is an electronic reprint of the original article. This reprint may differ from the original in pagination and typographic detail.

Author(s): Räsänen, Aleksi; Rusanen, Antti; Kuitunen, Markku; Lensu, Anssi

Title: What makes segmentation good? A case study in boreal forest habitat mapping

Year: $\quad 2013$

Version:

Please cite the original version:

Räsänen, A., Rusanen, A., Kuitunen, M., \& Lensu, A. (2013). What makes segmentation good? A case study in boreal forest habitat mapping. International Journal of Remote Sensing, 34(23), 8603-8627.

https://doi.org/10.1080/01431161.2013.845318

All material supplied via JYX is protected by copyright and other intellectual property rights, and duplication or sale of all or part of any of the repository collections is not permitted, except that material may be duplicated by you for your research use or educational purposes in electronic or print form. You must obtain permission for any other use. Electronic or print copies may not be offered, whether for sale or otherwise to anyone who is not an authorised user. 

What makes segmentation good? A case study in boreal forest habitat mapping

3 Aleksi Räsänen*, Antti Rusanen, Markku Kuitunen and Anssi Lensu Department of Biological and Environmental Science, University of Jyväskylä, Jyväskylä, Finland

6 *Corresponding author. Department of Biological and Environmental Science, P.O. Box

7 35, FI-40014 University of Jyväskylä, Email: t.aleksi.rasanen@jyu.fi 


\title{
What makes segmentation good? A case study in boreal forest habitat mapping
}

\author{
Segmentation goodness evaluation is a set of approaches meant for deciding \\ which segmentation is good. In this study, we tested different supervised \\ segmentation evaluation measures reviewed by Clinton et al. (2010) and visual \\ interpretation in the case of boreal forest habitat mapping in Southern Finland. \\ Used data were WorldView-2 satellite imagery and LiDAR digital elevation \\ model (DEM) and canopy height model (CHM) in 2-m resolution. Tested \\ segmentation methods were Fractal Net Evolution Approach (FNEA) and IDRISI \\ watershed segmentation. Overall, 252 different segmentation method, layer and \\ parameter combinations were tested. We also used eight different habitat \\ delineations as reference polygons against which 252 different segmentations \\ were tested. The ranking order of segmentations depended on the chosen \\ supervised evaluation measure; hence, no single segmentation could be ranked as \\ the best. In visual interpretation, we found out that several different \\ segmentations were rather good and selected one of them as the best. In \\ literature, it has been noted that better segmentation leads to higher classification \\ accuracy. We tested this argument by classifying 12 of our segmentations with \\ the random forest classifier. It was found out that there is no straightforward \\ answer to the argument, since the definition of good segmentation is inconsistent. \\ Highest classification accuracy $(0.72)$ was obtained with segmentation which was \\ regarded as one of the best in visual interpretation. However, almost as high \\ classification accuracies were obtained with other segmentations. We conclude \\ that one has to decide what she/he wants from segmentation and use \\ segmentation evaluation measures with care.
}

\section{Introduction}

Since the early 2000s, with the rise of object-based image analysis (OBIA) methodology (Blaschke 2010), segmentation goodness evaluation has been an emerging topic within the remote sensing literature (Clinton et al. 2010; Marpu et al. 2010). Evaluation has been concentrated on segmentation method development and comparison as well as parameter optimization.

Generally in segmentation, the goal is to partition imagery into regions that are meaningful; and thus, either mimic real world objects (Zhang, Fritts, and Goldman 2008; Clinton et al. 2010) or minimize intrasegment and maximize intersegment heterogeneity (Zhang, Fritts, and Goldman 2008; Hou et al. 2013). Remote sensing segmentation methods are a special case of more general image segmentation methods, which can be divided into two complementary groups: similarity or region based segmentation and discontinuity based segmentation. In region based segmentation, a similarity measure is used to find suitable regions. In discontinuity based segmentation, discontinuities of the images, usually boundaries, are detected (Zhang 1997; Gonzales and Woods 2002). Some of the methods combine concepts from both groups. For instance, in watershed segmentation, dividing lines between basin areas are sought by flooding the image (Gonzales and Woods 2002).

Within remote sensing, many of the segmentation implementations have been region based. Arguably, the most widely used remote sensing segmentation method has 
been Fractal Net Evolution Approach (FNEA) developed by Baatz and Schäpe (2000), and implemented in eCognition software. FNEA has been used as a benchmark segmentation, against which other methods have been compared. Although some authors have claimed to have developed better methods (Derivaux et al. 2010; H. Li et al. 2010; N. Li, Huo, and Fang 2010; Z. Wang, Sousa, and Gong 2010), FNEA has been a good performer in method comparisons (Neubert and Meinel 2003; Meinel and Neubert 2004; Carleer, Debeir, and Wolff 2005; Neubert, Herold, and Meinel 2008; Marpu et al. 2010) and seems to still be the standard method (e.g. Bar Massada et al. 2012; Duro, Franklin, and Dubé 2012). Also many other standard remote sensing analysis products such as ENVI, ERDAS Imagine and IDRISI Selva have included segmentation methods in their newer versions. Yet, there are also numerous other methods, algorithms and software applications for segmenting remotely sensed data. To analyse the goodness of these methods, segmentation evaluation has been performed (Zhang 1996; Clinton et al. 2010; Marpu et al. 2010).

Segmentation evaluation can be divided into two major categories: subjective (visual) and objective evaluation. Objective evaluation can be further divided into system-level, which evaluates the overall system in which segmentation is performed, and direct evaluation (Zhang, Fritts, and Goldman 2008). As an example, final classification output can be regarded as a system when assessing segmentation quality (sensu H. Li et al. 2010; Smith 2010; Z. Wang, Sousa, and Gong 2010; Gao et al. 2011). Direct evaluation can be either analytical or empirical, of which the former evaluates the method itself and the latter its results. Empirical methods consist of supervised and unsupervised methods, i.e. if ground truth is used as a reference or not (Zhang, Fritts, and Goldman 2008).

In remote sensing, analytical methods (Hay et al. 2003) and unsupervised methods (Espindola et al. 2006; Corcoran, Winstanley, and Mooney 2010; Drăguţ, Tiede, and Levick 2010; Yue et al. 2012; Hou et al. 2013) have been used in segmentation evaluation. For instance, Corcoran, Winstanley, and Mooney (2010) evaluate segmentation goodness by measuring contrast between segments that share a boundary. However, evaluation has largely been performed using supervised methods, more specifically either area based or location based measures. From these two, area based measures evaluate either if segmentation is too coarse (undersegmentation) or too fine (oversegmentation). Over- and undersegmentation measures can also be combined. Location based measures, on the other hand, are based on distances between segment centroids and reference polygon centroids or distances between boundary pixels. For a good review of these measures and an evaluation of different measures, see Clinton et al. (2010). These goodness measures are applicable especially in mapping clearly bordered urban features (Tian and Chen 2007; Zhan et al. 2005; Weidner 2008; Clinton et al. 2010), agricultural areas (Lucieer and Stein 2002; Möller, Lymburner, and Volk 2007; Z. Wang, Sousa, and Gong 2010) or larger land use / land cover types (Weidner 2008). Yet, goodness measures have also been used in natural area segmentations (Carleer, Debeir, and Wolff 2005; Ke, Quackenbush, and Im 2010; Bar Massada et al. 2012). Some of the supervised segmentation evaluation methods use a larger set of reference polygons inside a larger area (e.g. Clinton et al. 2010) whereas some of the methods use only a couple of distinct reference polygons and semi-automated approaches (e.g. Marpu et al. 2010).

Segmentation evaluation can be used to compare different types of segmentation methods, i.e. boundary against region-based (Carleer, Debeir, and Wolff 2005). As well, 
102 evaluation can be made between different segmentation methods or software, or inside a 103 segmentation method as parameter optimization (Marpu et al. 2010). Although similar 104 methods can be used in all these problems, also task-specific methodology for the 105 problems has been developed, especially to parameter optimization. For instance, 106 genetic algorithms have been used in optimizing segmentation to match reference 107 delineation (Feitosa et al. 2006; Chabrier et al. 2008). Optimization has also been made 108 without supervised goodness measures based on unsupervised evaluation. For instance, 109 Drăguţ, Tiede, and Levick (2010) developed a scale parameter optimization tool which 110 measures rate of change of local variance inside a scene. Optimal scale parameters are those which have local maximum of the rate of change. In a bit similar vein, Espindola et al. (2006), Gao et al. (2011), and Yue et al. (2012) tried to combine low intrasegment variance and low intersegment autocorrelation. On the other hand, Kim, Madden, and Warner $(2008,2009)$ hypothesized that optimal scales should only have low spatial autocorrelation between segments; whereas L. Wang, Sousa, and Gong (2004) maximized Battacharya distance between candidate segments. Finally, Smith (2010) optimized segmentation scale by minimizing classification error in a random forest classifier.

Forest inventory or forest habitat mapping is only one instance of where segmentation is often used. Yet, forest inventories are more and more dependent on automatic segmentations (Pekkarinen 2002; Hay et al. 2005; Castilla, Hay, and RuizGallardo 2008; Mustonen, Packalén, and Kangas 2008; Wulder et al. 2008; Falkowski et al. 2009; Kim, Madden, and Warner 2009; Ke, Quackenbush, and Im 2010; Hou et al. 2013). Segmentations used in forest inventory are usually made with feature values calculated from aerial or satellite images; however, the usage of light detection and ranging (LiDAR) data has recently become popular (Mustonen, Packalén, and Kangas 2008; Ke, Quackenbush, and Im 2010; Breidenbach et al. 2011; Eysn et al. 2012; Hou et al. 2013). It has been noted that synergy of imagery and LiDAR provide promising segmentation results and the selection of input data has an effect on segmentation quality (Geerling et al. 2007, 2009; Mustonen, Packalén, and Kangas 2008; Ke, Quackenbush, and Im 2010; Hou et al. 2013). Despite of this, studies incorporating different datasets remain scarce; both in forest inventory and in other applications.

In forest inventory or habitat mapping, segmentation goodness evaluations have been performed both qualitatively (Leckie et al. 2003; Wulder et al. 2008) and quantitatively using unsupervised (Kim, Madden, and Warner 2008, 2009; Hou et al. 2013) or supervised methods (Radoux and Defourny 2007; Ke, Quackenbush, and Im 2010). Some evaluations have been based on thematic quality of segments against reference polygons (Pekkarinen 2002; Mustonen, Packalén, and Kangas 2008). Wulder et al. (2008) criticize quantitative evaluation because used ground truth is a subjective delineation of forest patches; hence, real truth does not exist. Therefore, objects in forests are not as clearly separable as e.g. urban features. Furthermore, also in urban features, supervised evaluation has been criticized due to inaccurate ground truth (Corcoran, Winstanley, and Mooney 2010). In this work, we wanted to test if supervised segmentation evaluation methods are applicable to forested areas on a larger set of reference polygons.

It has been stated and tested that segmentation goodness affects directly classification accuracy in OBIA classification (Kim, Madden, and Warner 2009; Clinton et al. 2010; Ke, Quackenbush, and Im 2010; Gao et al. 2011). Although there are many different approaches and measures for segmentation goodness evaluation, the 
evaluation of goodness measures has not been thorough. In this paper, we will test if

151 widely used supervised segmentation goodness measures are applicable in boreal forest

152 habitat type mapping and if best segmentation leads to best classification accuracy. We

153 test supervised methods instead of unsupervised methods, because our goal is to find a

154 segmentation which matches with habitat type patches that are delineated using field

155 work. Furthermore, we try to find a segmentation method, parameter value and

156 image/data layer combination which suits our purposes. To do this, we test two different

157 methods (FNEA region based segmentation and IDRISI watershed segmentation),

158 several parameter combinations and different layers derived from WorldView-2

159 imagery and LiDAR data. We also test if different methods are habitat type, reference

160 polygon, or area sensitive.

\section{Methods}

162

163

164

165

166

167

168

169

170

171

172

173

174

175

176

177

178

179

180

181

182

183

184

185

186

187

188

189

190

191

192

193

\subsection{Study area and reference polygons}

We studied a $7 \mathrm{~km}^{2}$ rural-forested area southwest of the city of Jyväskylä located in Southern Finland. The area belongs to southern boreal vegetation zone (Ahti, HämetAhti, and Jalas 1968). The geographic coordinates (WGS84) of the site are $62^{\circ} 10^{\prime} 30^{\prime \prime}-$ $62^{\circ} 13^{\prime} 30^{\prime \prime} \mathrm{N}$ and $25^{\circ} 29^{\prime} 0^{\prime \prime}-25^{\circ} 38^{\prime} 0^{\prime \prime}$ E. The study area mainly consists of both coniferous and deciduous forest habitats, mires and agricultural area. The main tree species of the study area are the Scots Pine (Pinus sylvestris), Norwegian Spruce (Picea abies) and Birches (Betula pubescens and B. verrucosa). The study area was divided into three sub-areas with slightly varying land cover. The sub-areas were classified into 25 different habitat types (Table 1), which were mapped by field work during JuneAugust 2011. Habitat patches were used as reference polygons in segmentation goodness measures.

(Table 1 should be inserted here)

Most of the studied forest area is under heavy forestry and clear-cuts which create a human induced dynamic. Yet, two of the three delineated sub-areas included also one protected area which covered 100 ha and 25 ha of these sub-areas. Protected areas were dominated by semi-natural, over 100-year-old, forest. The larger protected area is part of a NATURA 2000 area. Inside the NATURA area and our study area, several different NATURA 2000 habitats are found. NATURA 2000 habitats were not mapped per se, but they were included in some of our mapped habitat types.

The three studied sub-areas were selected from different parts of a larger area southwest of Jyväskylä so that they included many different habitat types and different landscape configurations. Each sub-area was delineated into habitat patches whom there were 628 in total. Sub-area 1 (Sallaajärvi) included small to medium sized patches of different age, mostly mesic forest, some spruce mires, small streams, meadows, lakes and yards. In the area, there are also some old fields, which have been afforested. Moreover, in the middle of the area, there is a 250 ha conservation area with seminatural forest. Sub-area 2 (Kuusimäki) included large areas of protected old mesic forest with spruce mire patches. As well, this sub-area had some open and pine mires, small lakes and fields, yards, and different aged forest around the old forest. Sub-area 3 (Lapinmäki) included areas of bare rock surrounded by mesic forest, and with yards, fields and lakes on the fringes. 


\subsection{Remotely sensed data}

210 Our primary data consisted of 8-band multispectral 2-meter resolution WorldView-2

211 (WV-2) satellite image taken in July $14^{\text {th }} 2010$ and LiDAR data with a minimum of 0.5 212 points per $1 \mathrm{~m}^{2}$ from May 2010. Additionally, we used $20 \mathrm{~cm}$ resolution aerial images 213 (orthophotos) taken in 2007 in assisting the drawing of reference polygons.

214 WV-2 image, taken by Digital Globe Inc., consists of 8 bands: coastal blue

215 (band 1, 400-450 nm), blue (2, 450-510 nm), green (3, 510-580 nm), yellow $(4,585-$

$216625 \mathrm{~nm})$, red $(5,630-690 \mathrm{~nm})$, red-edge $(6,705-745 \mathrm{~nm})$, NIR1 (7, 770-895 nm), NIR2

$217(8,860-1040 \mathrm{~nm})$ in 2 meter resolution and a panchromatic band (450-800 nm) in 50

$218 \mathrm{~cm}$ resolution. Image was delivered radiometrically and sensor corrected, projected to a

219 plane with average terrain elevation. In our preprocessing phase, the image was first

220

221

222

223

224

225

226

227

228

229

230

231

232

233

234

235

236 orthorectified using 5-meter resolution digital elevation model derived from LiDAR data. In georeferencing, 13 ground control points from block features (buildings etc.) which were scattered all over study area were taken from orthophotos and nearest neighbour sampling was used. In visual interpretation, the differences between orthophotos, LiDAR data and orthorectified WV-2 were at maximum a couple of meters. From WV-2, we used all multispectral bands in $2 \mathrm{~m}$ resolution.

LiDAR data was created by National Land Survey of Finland. Flying altitude is on average 2000 meters. Used scan angle was $\pm 20^{\circ}$ and laser pulse footprint on the ground approximately $50 \mathrm{~cm}$. Mean error in elevation information is at maximum 15 centimetres and in planar information at maximum $60 \mathrm{~cm}$. Data was delivered automatically classified to ground hits, low vegetation hits, low error hits and unclassified hits.

LiDAR point clouds were first triangulated and after that rasterized using LASTOOLS (Isenburg 2011). We first derived two layers in $2 \mathrm{~m}$ resolution from LiDAR: digital terrain model (DTM) and digital surface model (DSM). In DTM, only ground hits were used whereas in DSM, point cloud was first thinned to one meter resolution to include highest hits. Then we subtracted DTM from DSM to create canopy height model (CHM). CHM was further manipulated to include values only between 0 and 40 meters to filter out unrealistic values. The CHM still had some wrong values below 40 meters but these could not be corrected easily. 
From DTM, we calculated also Saga Wetness Index (SWI) in 2 m resolution using SAGA-GIS to model soil moisture; and thus, potential places for mires. SWI is a modification of topographic wetness index (TWI). It has been noted that in wetland mapping standard TWI performed worse than some other models; however, mainly because in these studies TWI underestimated extent and contiguity of wetlands (Grabs et al. 2009, Murphy, Ogilvie, and Arp 2009). This might be due to that the standard TWI concentrates large values to stream networks where water flow is concentrated. This underestimation problem is overcome in SWI, which assumes homogenous hydrologic conditions in flat areas and predicts larger moisture values for cells with small vertical distance to streams (Böhner and Selige 2006, Equations 1 and 2).

$$
\alpha_{M}=\alpha_{\max } t^{-\beta \exp \left(t^{\beta}\right)} \text { for } \quad \alpha<\alpha_{\max } t^{-\beta \exp \left(t^{\beta}\right)}
$$

Specific catchment area $(\alpha)$ used in TWI is defined as the pixels upslope contributing area per contour width whereas $\alpha_{M}$ is modified catchment area used in SWI. In calculating $\alpha$, slope angle $\beta$ (in radians) and neighbouring cell maximum $\alpha_{\max }$ are taken into account unless results remain unchanged. Parameter $t$ is a value for suction, so that lower values, e.g. under 10, lead to stronger suction and stronger spreading of large $\alpha$ values, and higher values lead to weaker suction. After counting $\alpha_{M}$, SWI is calculated with the standard equation given in Equation (2).

$$
\mathrm{SWI}=\ln \left(\frac{\alpha_{M}}{\tan \beta}\right)
$$

Before calculations, DTM was filled to remove uncertainties, missing values and false values from the data. Before the filling, values in DTM in known and evident places of bridges and culverts were manipulated to let imagined water to flow through road banks in those locations. To angle $\beta, 0.0174532$ rad was added so that division by 0 was avoided. In flow direction calculations, we used multiple flow direction method by Freeman (1991). In this method, the slope value is raised to the power of 1.1. Thus, steeper slopes are weighted only a bit. It has been noted that in relatively flat areas multiple flow direction methods, in which slope value is raised by a low exponent (e.g. 0.5 to 2), give good results in TWI calculation (Güntner, Seibert, and Uhlenbrook 2004; Sørensen, Zinko, and Seibert 2006; Kopecký and Čížková 2010). Furthermore, parameter $t$ in Equation 1 was decided to be default 10 after visual interpretation of SWI with different $t$ values.

Before segmentation, SWI was quantized to 32 classes using equal intervals and $\mathrm{CHM}$ was quantized to 40 classes (nearest integer). WV-2 layers were first filtered using a $3 \times 3$ window and a median filter. After filtering, layers were quantized to 256 classes.

\subsection{Segmentation methods}

Data was segmented using different datasets, methods and parameters. To compare different types of segmentation methods, two segmentation methods were used: one watershed segmentation and one region based segmentation method. Next, brief introductions of the used segmentation methods and their parameters are given.

Watershed segmentation was implemented in IDRISI Taiga software. In IDRISI segmentation, a variance image is derived from each layer by moving window analysis. 
A weighted average of variance images is the final surface image for watershed delineation. Both the size of the moving window as well as the weights of averaging can be adjusted by the user. The values of this surface image are treated as elevation values like in a DEM, and pixels are grouped into watersheds. After watershed delineation, watersheds are merged iteratively. Pairs of segments are merged if they are most similar segments to each other in the neighbourhood and if their difference is smaller than a similarity tolerance adjusted by the user. Difference is evaluated by two aspects: the mean value and the standard deviation. The weights for the mean and for the standard deviation are set by the user.

Our region based segmentation was the widely used segmentation method of eCognition software, Fractal Net Evolution Approach (FNEA) (Baatz and Schäpe 2001; Benz et al. 2004). FNEA segmentation was carried out using TerraLib 4.2.0 C++-GISlibrary (Câmara et al. 2008). In FNEA, regions are formed by merging pixels; i.e. in the beginning, each pixel is treated as a region. In segmentation, three user parameters can be adjusted: scale parameter and weights between colour and shape $\left(w_{\text {color }}+w_{\text {shape }}=\right.$ $1)$ as well as smoothness and compactness $\left(w_{\text {smooth }}+w_{\text {compt }}=w_{\text {shape }}\right)$. Scale parameter controls the average object size. The more weight is given to colour (or spectral) homogeneity, the less weight is given to a specific shape i.e. spatial homogeneity. Smoothness and compactness define the shape as follows. Smoothness is the ratio of the border length of the segment and border length of the bounding box of the segment. Compactness, on the other hand, is the ratio of the border length of the segment and the square root of the number of pixels in the segment. Hence, they are not antagonistic but the weight is defined between them. Finally, the weights for the different layers are set by the user.

\subsection{Initial work for segmentation goodness evaluation}

In segmentation, several issues affect the final segmentation goodness: segmentation method, parameterization including weights for the layers (e.g. Marpu et al. 2010), used layers (e.g. Ke, Quackenbush, and Im 2010), (re)classification of the layers, transformations made for the layers and filtering of the layers (e.g. Carleer, Debeir, and Wolff 2005). Easily thousands of different combinations can be tested. Therefore, we first did initial trial-and-error testing and visual interpretation for different types of segmentation. We segmented single layers, reclassified and filtered the layers and tried different parameter combinations and segmentation methods. In our initial analysis, the goal was to find good segmentation methods that could be further evaluated using the evaluation measures. As well, we wanted to scale our layers so that they could be used in same segmentations; in other words, the segments that are produced in single layer segmentations should be approximately of same size. Needless to say, our initial evaluation was not thorough but it was good enough to find good segmentation methods. All possible combinations could not be tested but we found a set of segmentations that were probably among the best that are available.

\subsection{Used parameter and layer combinations}

Four different layer combinations were tested: (a) WV-2 layers only, (b) LiDAR layers only, (c) WV-2 bands 2, 3, 5, 7 (blue, green, red, NIR1) and LiDAR layers, and (d) all layers. IDRISI segmentation was performed using a window size of 5. Similarity 
tolerance was varied between 20 and 70 with intervals of 5 . Three different combinations of mean and variance weights were used: mean 0.5 , variance 0.5 ; mean 0.9 , variance 0.1 ; and mean 0.1 , variance 0.9 . Hence, overall 33 IDRISI segmentations were performed for all layer combinations. FNEA segmentation was performed by varying the scale parameter between 5 and 50 with intervals of 5 , and using colour parameter values of $0.25,0.5$ and 0.75 . Therefore, 30 different FNEA segmentations were done for all layer combinations. In all segmentations, all layers were given equal weight.

\subsection{Different reference polygons}

We tested different segmentation methods using eight different reference polygon sets. First, all reference polygons from the whole study area were used. Second, three sets included all reference polygons from three different sub-areas separately. Third, two sets included reference polygons of only one habitat type: one set included all mires and one set water. Finally, we tested segmentation quality against two other reference polygon sets (FFPS biotope and forestry planning data) (Figure 1).

(Figure 1 should be inserted here)

\subsection{Goodness evaluation measures}

Segmentation goodness was evaluated using several different supervised measures (Table 2) reviewed by Clinton et al. (2010) with a Java tool that they developed. For more clarification and equations, please refer to Clinton et al. (2010) and original publications listed in Table 2. All measures were calculated as a mean of all reference polygons inside a reference polygon set. The value for a specific reference polygon was calculated as a mean (or standard deviation) of the values of those segments that met at least one out of four criteria. Criteria were: (1) the centroid of the segment is inside the reference polygon, (2) the centroid of the reference polygon is inside the segment, (3) the shared area of the segment and the reference polygon is over 0.5 of the segment area, and (4) the shared area of the segment and the reference polygon is over 0.5 of the reference polygon area (Clinton et al. 2010). Some of the measures were weighted by the reference objects (Table 2). Furthermore, we calculated combined measures which were proposed by Clinton et al. (2010) and which all included measures from single authors only (Table 3). Some of the combined measures were calculated as root mean square (RMS) individual criterion values whereas some of them were simple sum calculations. In RMS calculations, all measures were adjusted so that ideal segmentation was set to 0. Finally, a combined measure COMBINED was calculated which was a RMS of all basic area and location-based measures as suggested by Clinton et al. (2010). However, QLoc was not included since it was the same measure as RPsub. Before RMS calculation in COMBINED, all measures were scaled to $[0,1]$ by dividing each value with the maximum and setting ideal segmentation to 0 .

(Tables 2 and 3 should be inserted here)

Furthermore, we measured segmentation goodness using visual interpretation. In visual interpretation, we paid detail especially to if the segmentation methods find the boundaries of some reference polygons and habitat types. Hence, we were more worried about undersegmentation than oversegmentation. Additionally, we checked if different kinds of habitat types are segmented and if the segmentation produces objects that are 
meaningful entities and can be easily used in classification and planning (Hay et al.

371 2005). Therefore, segments should not be too complex (Mustonen, Packalén, and

372 Kangas 2008). Due to the large number of different segmentations, our visual

373 interpretation was not thorough; instead, we tried to find some general trends from

374 different segmentation methods as well as layer and parameter combinations.

\section{$375 \quad 2.8 \quad$ Classifications}

376 After segmentation evaluation, we selected 12 segmentations for classification.

377 Segmentations were selected using subjective evaluation; so that meaningful evaluation

378 of segmentation performance versus classification accuracy could be made and some of

379 the segmentations could be compared to each other. Both good and not as good

380 segmentations, based on evaluation measures and visual interpretation, were selected. In

381 classification, we calculated mean values of each layer per segment. In all

382 classifications, all layers were always used regardless of which layer combination a-d

383 was used in the segmentation phase.

384 Supervised classification was performed using the random forest classifier

385 (Breiman 2001) with R package randomForest (Liaw and Wiener 2004) in R version

386 2.15.2 (R Development Core Team 2012). Random forest classification has been used

387 in remote sensing and OBIA with good experience (Lawrence, Wood, and Sheley 2006;

388 Rodriguez-Galiano et al. 2012). Random forest is an ensemble classifier, which

389 combines several bootstrapped classification trees. In the final classification majority

390 vote over all trees is made. Trees are randomized at each node by selecting only a subset

391 of variables of which the best split is chosen. When a tree is built, approximately $2 / 3$ of

392 the data is selected for training the classifier and the rest is called out of bag (OOB) test

393 data. OOB data is used for error rate estimation, which is averaged over all trees to get

394 an error rate for the whole classification. Because of the OOB, independent test data or

395 cross-validation is not needed when random forest is used (Breiman 2001; Breiman and

396 Cutler 2007) which has been confirmed in remote sensing studies (Lawrence, Wood,

397 and Sheley 2006; Rodriguez-Galiano et al. 2012).

398 When random forest was performed, 500 trees were built and the number of

399 features at each split was given the default value of square root of all features. We used

400 our own reference polygons over all three sub-areas as training data, not the FP nor the

401 FFPS data. Training set in random forest run was all those segments that had a

402 minimum of $60 \%$ coverage of one reference habitat type. Classification accuracies

403 were calculated using all reference polygons with simple cross-tabulation matrices.

\section{$404 \quad 3 \quad$ Results}

\subsection{Segmentation goodness based on evaluation measures}

406 Based on all area and COMBINED measure, best segmentation was FNEA with layer

407 set b, scale parameter 25 and colour parameter 0.5 (Table 4, Table 5). However,

408 choosing this segmentation as the best was contradictory, since no other segmentation

409 evaluation measure ranked it as the best method. As well, its rank was between 4 and 94

410 when COMBINED measure and other reference polygons than all area were used.

411 Hence, different segmentations were chosen as the best or being among the best, when

412 different goodness measures or reference polygons were used (Table 4). Some of the 
413 measures (UnderMerging, OverMerging, CountOver, SimSize sd, RAsuper, RAsub, 414 OverSegmentation, UnderSegmentation), nonetheless, gave rather consistent results,

415 i.e., the same segmentation was the best or one of the best using different reference

416 polygon sets. Other measures, on the other hand, had larger variation in their results.

417 Consistency in results can be seen as a downside, since reference sets were different as

418 illustrated in Figure 1. For instance, individual measures may prefer segmentation

419 result, which is as fine or as coarse as possible. On the other hand, consistency can also

420 be seen as an asset if some of the segmentations truly are better despite of the reference

421 set used, i.e. those segmentations contain almost all meaningful patch boundaries.

422

423

424

425

426

427

428

429

430

431

432

433

434

435

436

437

438

439

440

441

442

443

444

445

446

447

448

449

450

451

(Tables 4 and 5 should be inserted here)

Some overall evaluations can be made from segmentations ranked as the best

(Table 6). First, FNEA segmentation outperformed IDRISI segmentation, since FNEA was ranked best 140 times against 44 times of IDRISI. Second, layer set b outperformed other layer sets. Therefore, it could be thought that FNEA with layer set $b$ provides the best results. If undersegmentation is wanted to be avoided, low scale parameter brings good results. Vice versa, high scale parameter should be selected when oversegmentation is not desired. Segmentations with intermediate scale or similarity parameter value were not ranked as best as often as segmentations with high or low parameter value. Yet, different combined measures as well as AFI, RP measures, SimSize mean, QLoc mean, QLoc sd and QR usually preferred intermediate scale parameter values. For instance, when all area reference polygons were used, the COMBINED measure favoured intermediate scale parameter values; whereas it ranked those segmentations with low scale parameter value as the worst (Table 5). In FNEA segmentations, high value for colour parameter gave more often best segmentations than low or intermediate value for colour. In IDRISI segmentations, on the other hand, high mean, low variance combination gave the largest number of best segmentations.

(Table 6 should be inserted here)

When correlations between different goodness measure results were evaluated (Table 7), it was found out that correlations range from large negative correlations to high positive correlations. Hence, measures did give different results and preferred different issues in segmentation. It can also be seen that measures that measure oversegmentation had positive correlations with the COMBINED measure whereas undersegmentation measures had negative correlations (for over- and undersegmentation measures, see Table 2). Some measures (RPsuper, MergeSum, M, $\mathrm{ZH} 1$ ) had even both positive and negative correlations. Correlations were dependent on reference polygons used; but correlations between the COMBINED measure based on different reference polygons were rather high and positive (Table 8). Only water has correlations below 0.75 .

(Tables 7 and 8 should be inserted here)

\subsection{Segmentation goodness based on visual interpretation}

453 In visual interpretation, it was found out that segmentations based on layer set $b$

454 (LiDAR data only) were especially successful in delineating mires and small streams.

455 Also the problem of shadow effect in WV-2 imagery was overcome when LiDAR data

456 was used. On the other hand, the shorelines of water bodies were insufficiently

457 delineated with LiDAR data only. As well, boundaries between deciduous and

458 coniferous forests were better delineated using WV-2 imagery. However, more gradual 
459 boundaries, for instance between mesic and xeric forests, could not be easily segmented

460 using any method or layer combination. In visual interpretation, we could not make a

461 preference between layer sets $\mathrm{c}$ and $\mathrm{d}$. Although segmentation outputs were slightly

462 different, differences were minor. Same kinds of observations were made, when

463 different IDRISI mean/variance weight alternatives were compared. Furthermore, to

464 delineate some small objects, small values of scale or similarity parameters were

465 needed. In finding meaningful and simple entities, it was found out that FNEA

466 segmentation with low (0.25) or intermediate (0.5) weight for colour brought superior

467 results over other segmentation methods. Putting little weight to colour had its

468 downside, on the other hand. In other words, segment boundaries did not necessarily

469 follow natural or data boundaries but segments were equally sized objects with often

470 arbitrary boundaries. Nevertheless, FNEA segmentations with large weight for colour

471 and IDRISI segmentations were unnecessarily complex. Additionally in IDRISI

472 segmentations, boundaries were often crisscrossing reference polygon boundaries.

473 Using visual interpretation, we chose FNEA segmentation with layer combination c,

474 scale parameter 10 and colour parameter 0.5 , as the best one (Figure 2c). This selection

475 was, yet, more or less arbitrary, since many different segmentation options gave quite

476 similar results. Furthermore, since there were so many different segmentation options,

477 visual interpretation was not thoroughly reliable in finding the best parameter values.

478 Hence, choosing the best segmentation using visual interpretation was tricky.

479

480

481

482

483

484

485

486

487

488

489

490

491

492

493

494

495

496

497

498

499

500

501

502

503

\subsection{Classification results}

Classification accuracies between classifications derived from different segmentations varied a bit (Table 9, some of the segmentations in Figure 2). Best accuracy (0.72) was achieved using best segmentation in visual interpretation (Figure $2 \mathrm{c}$ ) whereas worst accuracy (0.60) was got using segmentation that was ranked high using some of the measures (Figure 2h). Many of the different segmentations got reasonably good results compared to the best classification. Some of these segmentations were selected based on measures, some by using visual interpretation. On the other hand, best segmentation based on COMBINED measure and all area (Figure 2e), was not among the best segmentations in classification accuracy analysis. It can be seen that fine or moderately fine segmentations led to better classification accuracies. Vice versa, coarse segmentation led to poorer accuracies. On the other hand, too fine segmentation can lead to salt-and-pepper effect (Figure $2 b$ ) and thus possibly also make classification accuracy worse. Also in visual interpretation it became evident that classifications performed with FNEA segmentations and scale parameter value 5 suffered from this effect more than classifications performed with segmentations with scale value 10 . Best classification accuracies were achieved using segmentations with both LiDAR and WV2 layers. This might be due to the fact that boundaries were best detected using both data types in segmentation. Yet, classification accuracies using only segmentations performed with LiDAR or WV-2 data were nevertheless little worse. In all, classification accuracy evaluation was not thorough; in other words, good classification accuracies can be got using segmentations, which were not among the 12 segmentations tested here. Moreover, some measures may be good in selecting segmentations that maximize classification accuracy.

(Table 9 and Figure 2 should be inserted here) 


\section{Discussion}

505

506

507

508

509

510

511

512

513

514

515

516

517

518

519

520

521

522

523

524

525

526

527

528

529

530

531

532

533

534

535

536

537

538

539

540

541

542

543

544

545

546

547

548

549

\subsection{Segmentation goodness compared to classification accuracy}

One of our study objectives was to test if better segmentation leads to better classification accuracy as it has been argued by others (Kim, Madden, and Warner 2009; Clinton et al. 2010; Ke, Quackenbush, and Im 2010; Gao et al. 2011). After our analysis, it is obvious that there is no straightforward answer to this question. Although it is self-evident that good segmentation is needed for good classification, there is no adequate definition of what makes a good segmentation. After classification analysis one can easily state that the best segmentation was the segmentation with the best classification accuracy. There is no method, however, to test before classification, which segmentation will give the best classification accuracy. This is illustrated also by the studies of Kim, Madden, and Warner (2009) and Gao et al. (2011). While they both claim that optimal segmentation produced best classification output, their definitions of optimal segmentation were contradictory. Kim, Madden, and Warner (2009) minimized spatial autocorrelation between different segments, whereas Gao et al. (2011) sought for segmentations that combined low intersegment autocorrelation and intrasegment variance. Furthermore, Gao et al. (2011) had the lowest intersegment autocorrelation at the coarsest scale which did not produce best classification accuracy. On the other hand, the tasks in these studies were different, since Kim, Madden, and Warner (2009) used 4$m$ resolution IKONOS data in forest type mapping, while Gao et al. (2011) used 25-m Landsat ETM+ data in mixed mountainous shrub-forest-grassland landscape.

Based on ambivalence of what segmentation is good, we propose that the goodness of segmentation should be defined in each case. In other words, one should know and clarify what he/she wants from segmentation; and critically evaluate if the best segmentation can be selected based on evaluation criteria. In our case, good segmentation was segmentation with (1) meaningful and not too complex segments, (2) boundaries parallel to reference polygon boundaries even for the smallest reference polygons but (3) as coarse as possible. Taking the best segmentation based on some measure does not automatically lead to the best classification accuracy, as it has been already noted by Verbeeck, Hermy, and van Orshoven (2012). Nevertheless, the classification accuracies between different classifications were rather small in our case study. This might point out to the robustness of OBIA methodology: good classification accuracy can be obtained even if the segmentation is not the best possible. On the other hand, the classification outputs that had better classification accuracies were visually more appealing. Boundaries were more often in right place, different habitat types could be mapped and patches were not too small.

Many authors have argued that oversegmentation is a smaller problem than undersegmentation in post-segmentation classification (e.g. Weidner 2008; Marpu et al. 2010). However, in the analysis by Verbeeck, Hermy, and van Orshoven (2012), it was found out that more under-segmented output gave better classification accuracy than more over-segmented output. Our results suggest that both arguments are partly correct. In other words, both oversegmentation and undersegmentation are problematic in classification as it was found out also by Kim, Madden, and Warner (2009) and Gao et al. (2011). First, segmentation cannot be very coarse, since smaller objects are thus easily under-segmented. Second, if segmentation is too fine, salt-and-pepper effect is obtained. Salt-and-pepper effect can lead to worse classification accuracy as it has been 
550 found out in OBIA vs. pixel-based classification studies (e.g. Bock et al. 2005;

551 Whiteside, Boggs, and Maier 2011). Also, objects are more meaningful when they are

552 not too small (Blaschke 2010). Yet, the better classification accuracy of OBIA is not

553 automatic and some smaller, but rare, objects may be easily missed in OBIA

554 classification (Dingle Robertson and King 2011). Overall, it has been noted that in

555 single-scale segmentation optimal segmentation is class-dependent, i.e., some classes

556 can be poorly segmented even if overall segmentation is optimal. Hence, multi-scale

557 segmentation has been offered as a solution to this problem (Hay et al. 2003; Kim et al.

558 2011; dos Santos et al. 2012).

559

560

561

562

563

564

565

566

567

568

569

570

571

572

573

574

575

576

577

578

579

580

581

582

583

584

585

586

587

588

589

590

591

592

593

594

595

\subsection{Object and patch delineation}

We found out that some habitat patches were not segmented properly using any of the methods, layers or parameter combinations. For instance stream-sided habitats or mires were often poorly delineated. Therefore, some extra analysis is needed, such as stream network mapping (Räsänen et al., in prep), other ancillary information or expert knowledge (Mustonen, Packalén, and Kangas 2008) or segmentation post-modification to delineate some of the patches correctly. It can even be asked, can the segmentation goodness over difficult patch delineation even be calculated. For instance, Radoux and Defourny (2007) delineated only those patches that could be seen from imagery. Therefore, it is not realistic to expect that segmentation delineates those objects that cannot be easily seen from the data that is segmented. LiDAR data, however, helped in finding some of the tricky features, such as mires. On the other hand, segmentations with LiDAR data and four WV-2 layers were not significantly different compared to segmentations with LiDAR data and eight WV-2 layers. As well, our study reasserted earlier studies that the problematic shadow effect of aerial or satellite imagery can be mitigated using LiDAR data (Geerling et al. 2007; Mustonen, Packalén, and Kangas 2008; Ke, Quackenbush, and Im 2010). Segmentations based on imagery only; nonetheless, produced classifications with almost as high classification accuracies as segmentations based on both imagery and LiDAR data. There can be at least two possible reasons for this small difference. First, segmentation based only on imagery may have other benefits compared to segmentation using both data types. Second, the proportion of shadow areas over all area can be rather small, especially with data resolution not higher than $2 \mathrm{~m}$.

One major question in segmentation evaluation is whether it is better to delineate meaningful objects with meaningful thematic quality and maximum homogeneity (e.g. Mustonen, Packalén, and Kangas 2008) or to find segmentation that mimics field observations. Some authors (Wulder et al. 2008; Corcoran, Winstanley, and Mooney 2010) have questioned the rationality of supervised segmentation evaluation, especially in natural environments. It is true that nature is not easy to interpret. Different mappers classify habitat patches differently and also delineate patch boundaries differently. Yet, according to Cherrill and McClean $(1995,1999)$ the former type of error was more common in habitat mapping in the UK. Nevertheless, boundaries are not easy to draw and their location depends on the study scale (Lang et al. 2010). In our analysis, there were differences between boundary locations when our field data was compared to either FP or FFPS data. There were some differences between optimal segmentations based on different reference polygons. These differences were mostly minor, and approximately same kinds of segmentations were preferred irrespective of 
596 the reference data. As well, correlations between COMBINED measures based on

597 different reference data were rather high (SA1 to FP 0.79 and SA2 to FFPS 0.89).

598 Furthermore, segmentation evaluation based on thematic quality is not unproblematic

599 either. Although the segmentation has good thematic quality, the segmentation

600 boundaries do not necessarily match with habitat type boundaries that exist in nature.

601 This can lead to difficulties in habitat classification, if it is performed, and eventually to

602 differences in planning decisions.

603 In a more general level, one can question if automated segmentation is worse

604 than manual delineation when it cannot find the boundaries that are manually

605 delineated. Delineations are different; that is true, but it is not straightforward to judge

606 either one of them better. For instance, automated delineation often produces more

607 complex objects. Complexity of the objects, however, can be both good and bad.

608 Although complexities hinder the usage in operational context, complexity can be

609 reduced using GIS techniques. Furthermore, complex boundaries can be even truer,

610 since natural boundaries are not always straight (Wulder et al. 2008). Therefore,

611 automated and manual delineations are two different interpretations and both of them

612 can be either good or bad depending on the segmentation method, mapper skills or the

613 operational context. In other words, question is not necessarily if one of them is correct

614 or incorrect but whether it is appropriate or inappropriate (Lang et al. 2010).

\section{$615 \quad 4.3$ How to evaluate and measure segmentation goodness?}

616 According to our analysis, the FNEA was better segmentation method than the

617 watershed segmentation in IDRISI Taiga. Still, also IDRISI's segmentation method

618 provided good results. As already noted earlier, FNEA has been a good performer in

619 segmentation evaluations and is a standard method in OBIA studies. However, we

620 cannot give any percentage or any other quantitative evaluation which indicates how

621 much better FNEA is compared to IDRISI contrary to values given e.g. by N. Li, Huo,

622 and Fang (2010). N. Li, Huo, and Fang (2010) classified different types of objects to

623 correctly delineated, acceptably delineated and wrongly delineated. From these

624 classifications, they calculated performances of different segmentations and also the

625 percentage difference of performance. In our framework, such quantitative difference

626 evaluation would be more or less artificial, since in our study different measures of

627 segmentation goodness gave different results. This inconsistency has also been noted by

628 Clinton et al. (2010). Partly this inconsistency can be explained in terms of over- and

629 undersegmentation; i.e., deliberately avoiding one of them results often in getting the

630 other. However, also evaluation measures that should quantify the same phenomenon

631 can give different results. One explanation to this is that we tested several different

632 segmentations of which some were rather similar to each other. Furthermore, measures

633 are a bit dependent on the training data set used. One should, thus, be careful in the

634 selection of the reference data. On the other hand, some of the measures were robust,

635 i.e. produced similar results irrespective of the reference data. Additionally, general

636 picture was more or less similar with different reference polygons.

637 According to classification accuracies derived in our study, the best

638 segmentation was found using visual interpretation. Therefore, it could be argued that

639 supervised segmentation goodness evaluation measures evaluated by Clinton et al.

640 (2010) are not good. On the other hand, we knew what we wanted from visual

641 interpretation and fixed our objectives based on these needs. Automated supervised 
642 segmentation goodness evaluation measures, on the contrary, were just selected based

643 on what has been done before. Therefore, we knew better what we want from

644 segmentation when we evaluated them visually: meaningful objects and boundaries.

645 Yet, we would have included our own automated and supervised evaluation method in

646 our analysis, if we had found a successful way to do automated evaluation. One reason

647 that supervised segmentation goodness evaluation measures partly failed in our analysis

648 could be that we used continuous reference polygon data and objects that were difficult

649 to delineate. On the other hand, segmentation goodness measures did not work that well

650 on water bodies either although water bodies are usually easy to delineate and are not

651 bordered by each other. Based on measures, best segmentations for water bodies were

652 usually segmentations using LiDAR data or segmentations as fine as possible. In our

653 visual interpretation, it was, nonetheless, found out that water bodies cannot be

654 delineated using LiDAR data alone. Only evaluation measure M ranked best a

655 segmentation that could be good in water body delineation. Nevertheless, we cannot say

656 that supervised evaluation measures are completely useless. On the contrary, one should

657 know what evaluation measures favour and what she/he wants from segmentation

658 before using evaluation measures. As well, visual interpretation is subjective, tedious

659 and time-consuming (Zhang, Fritts, and Goldman 2008). It can be even practically

660 impossible if several different segmentations over large areas should be evaluated.

661 Automated segmentation goodness evaluation could be done using landscape or

662 shape metrics and thus unsupervised evaluation (Neubert and Meinel 2003, Meinel and

663 Neubert 2004, Neubert, Herold, and Meinel 2008, H. Li et al. 2010, Ji et al. 2012). This

664 is problematic, though, since for instance FNEA method uses shape metrics as

665 parameters which the user can modify. Hence, using shape metrics also in evaluation

666 could lead to circular reasoning. Another possible solution in finding good segmentation

667 evaluation measures could be focusing on boundaries. In other words, it could be

668 examined if boundaries drawn in the reference map are found in segmentation. For

669 instance, Neubert and Herold (2008) measured what proportion of segment's perimeter

670 is inside specific reference polygon's buffer zone. In similar vein, Lucieer and Stein

671 (2002) have proposed boundary based measure and Clinton et al. (2010) included a

672 modification of this measure in their analysis. Whereas Lucieer and Stein (2002)

673 calculated shortest distances from reference polygons to any boundary pixel in

674 segmentation, Clinton et al. (2010) averaged all distances to all segments inside

675 reference polygon. Of these measures, original measure by Lucieer and Stein (2002) is

676 more tempting, since boundaries inside reference polygon can disappear in

677 classification but boundaries near reference polygon boundaries cannot be moved.

678 However, Lucieer and Stein (2002) noted that finest segmentations ranked best using

679 this evaluation. Taking this into account, they modified the original measure to take the

680 length of boundary into account. These kinds of modifications, on the other hand, are

681 difficult to design, because they easily favour either undersegmentation or

682 oversegmentation. Furthermore, boundaries of natural objects are not exact. Hence, it is

683 not always meaningful to find the "real" boundaries but boundaries that are visible in

684 data.

685 Finally, unsupervised segmentation evaluation methods that often measure

686 intersegment and intrasegment homogeneity or heterogeneity have been found useful in

687 segmentation evaluation (Kim, Madden, and Warner 2009; Gao et al. 2011; Yue et al.

688 2012; Hou et al. 2013). While in our case the goal was to find segmentation that mimics 
689 reference polygons, it could be interesting to test if unsupervised methods work well in 690 this kind of task.

\section{Conclusion}

692 We tested different supervised segmentation goodness evaluation measures and visual

693 interpretation to find a good segmentation for boreal forest habitat mapping. While

694 different supervised segmentation goodness measures were fast to calculate from

695 several segmentations, they provided inconsistent results. In other words, different

696 segmentations stood out as being best when different measures were used. Visual

697 interpretation, on the other hand, was tedious and segmentations could not be evaluated

698 thoroughly in reasonable time. Although we selected only one segmentation as being

699 the best based on visual interpretation, also other segmentations were visually good. In

700 classification analysis, the visually selected segmentation gave the best classification

701 accuracy but differences between different segmentations were rather small. Better

702 segmentation may lead to better classification but there are several different definitions

703 for good segmentation. Therefore, the relationship between segmentation and

704 classification is not straightforward. We propose that the goodness of segmentation

705

706

707

708

709

710

711

712

713

714

should be defined in each case separately and evaluation measures should be selected

based on that definition. In our case, good segmentation was segmentation with (1)

meaningful and not too complex segments, (2) boundaries parallel to reference polygon

boundaries even for the smallest reference polygons but (3) as coarse as possible. There were, however, no evaluation measures to find these kinds of segmentations

automatically. Overall, the best segmentations were FNEA segmentations with both imagery and LiDAR data. We conclude that different segmentation evaluation methods should be used with care especially in natural environment mappings. When segmentation evaluation is rigorously used; though, it can assist in finding a more optimal segmentation. Quantitative segmentation evaluation might provide better results in urban environments but more thorough testing is needed to support this claim.

\section{Acknowledgements}

717 This research was funded by Maj and Tor Nessling foundation. We are grateful to City of

718 Jyväskylä for providing us the reference polygon dataset and the aerial imagery as well as to

719 Finnish Forest and Park Service for the reference polygon dataset. MK received from Jenny and

720 Antti Wihuri foundation a sabbatical scholarship for the year 2013 that was partly funded also

721 by the EU IMPERIA-project (LIFE11 ENV/FI/905) and the University of Jyväskylä.

\section{References}

Ahti, Teuvo, Leena Hämet-Ahti, and Jaakko Jalas. 1968. "Vegetation Zones and Their Sections in Northwestern Europe." Annales Botanici Fennici 5: 169-211.

Baatz, Martin and Arno Schäpe. 2000. "Multiresolution Segmentation - An Optimization Approach for High Quality Multi-Scale Image Segmentation.” In Angewandte Geographische Informationsverarbeitung XII, edited by J. Strobl and G. Griesebner, 12-23. Heidelberg: Wichmann.

Bar Massada, Avi, Rafi Kent, Lior Blank, Avi Perevolotsky, Liat Hadar, and Yohay Carmel. 2012. "Automated Segmentation of Vegetation Units in a 
Mediterranean Landscape.” International Journal of Remote Sensing 33 (2): 346-364.

Benz, Ursula C., Peter Hofmann, Gregor Willhauck, Iris Lingenfelder, and Markus Heynen. 2004. Multi-Resolution, Object-Oriented Fuzzy Analysis of Remote Sensing Data for GIS-Ready Information. ISPRS Journal of Photogrammetry and Remote Sensing 58: 239-258.

Blaschke, Thomas. 2010. "Object Based Image Analysis for Remote Sensing." ISPRS Journal of Photogrammetry and Remote Sensing 65: 2-16.

Bock, Michael, Panteleimon Xofis, Jonathan Mitchley, Godela Rossner, and Michael Wissen. 2005. "Object-Oriented Methods for Habitat Mapping at Multiple Scales - Case Studies from Northern Germany and Wye Downs, UK." Journal of Nature Conservation 13: 75-89.

Böhner, Jürgen, and Thomas Selige. 2006. "Spatial Prediction of Soil Attributes using Terrain Analysis and Climate Regionalisation." In SAGA - Analysis and Modelling Applications, edited by Jürgen Böhner, K. R. McCloy, and J. Strobl, 13-28. Göttinger Geographische Abhandlungen, Vol.115.

Breidenbach, Johannes, Erik Næsset, Vegard Lien, Terje Gobakken, and Svein Solberg. 2010. "Prediction of Species Specific Forest Inventory Attributes using a Nonparametric Semi-Individual Tree Crown Approach Based on Fused Airborne Laser Scanning and Multispectral Data." Remote Sensing of Environment 114: 911-924.

Breiman, Leo. 2001. "Random Forests." Machine Learning 45: 5-32.

Breiman, Leo, and Adele Cutler. 2007. "Random Forest: Classification Description." http://www.stat.berkeley.edu/ breiman/RandomForests/cc_home.htm.

Câmara, Gilberto, Lubia Vinhas, Karine Reis Ferreira, Gilberto Ribeiro de Queiroz, Ricardo Cartaxo Modesto De Souza, Antonio Miguel Vieira Monteiro, Marcelo Tílio de Carvalho, Marco Antonio Casanova, and Ubirajara Moura De Freitas. 2008. "TerraLib: An Open Source GIS Library for Large-Scale Environmental and Socio-Economic Applications." In Open Source Approaches in Spatial Data Handling, edited by G. Brent Hall, and Michael G. Leahy, 247-270. Berlin: Springer.

Carleer, A. P., O. Debeir, and E. Wolff. 2005. "Assessment of Very High Resolution Satellite Image Segmentations.” Photogrammetric Engineering \& Remote Sensing 71 (11): 1285-1294.

Castilla, Guillermo, Geoffrey J. Hay, and Jose R. Ruiz-Gallardo. 2008. “SizeConstrained Region Merging (SCRM): An Automated Delineation Tool for Assisted Photointerpretation." Photogrammetric Engineering \& Remote Sensing 74 (4): 409-419.

Chabrier, S., C. Rosenberger, B. Emile, and H. Laurent, H. 2008. "Optimization-Based Image Segmentation by Genetic Algorithms." EURASIP Journal on Image and Video Processing, Article ID 842029. doi:10.1155/2008/842029.

Cherrill, Andrew, and Colin McClean. 1995. An Investigation of Uncertainty in Field Habitat Mapping and the Implications for Detecting Land Cover Change. Landscape Ecology 10 (1): 5-21.

Cherrill, Andrew, and Colin McClean. 1999. The Reliability of 'Phase 1' Habitat Mapping in the UK: The Extent and Types of Observer Bias. Landscape and Urban Planning 45: 131-143. 
Clinton, Nicholas, Ashley Holt, James Scarborough, Li Yan, and Peng Gong. 2010. "Accuracy Assessment Measures for Object-based Image Segmentation Goodness." Photogrammetric Engineering \& Remote Sensing 76 (3): 289-299.

Corcoran, Padraig, Adam Winstanley, and Peter Mooney. 2010. "Segmentation Performance Evaluation for Object-Based Remotely Sensed Image Analysis". International Journal of Remote Sensing 31 (3): 617-645.

Derivaux, S., G. Forestier, C. Wemmert, and S. Lefévre. 2010. "Supervised Image Segmentation using Watershed Transform, Fuzzy Classification and Evolutionary Computation." Pattern Recognition Letters 31 (15): 2364-2374.

Dingle Robertson, Laura, and Douglas J. King. 2011. "Comparison of Pixel- and Object-Based Classification in Land Cover Change Mapping." International Journal of Remote Sensing 32 (6): 1505-1529.

dos Santos, Jefferson Alex, Philippe-Henri Gosselin, Sylvie Phillipp-Foliguet, Ricardo da S. Torres, and Alexandre Xavier Falcão. 2012. "Multiscale Classification of Remote Sensing Images". IEEE Transactions on Geoscience and Remote Sensing 50 (10): 3764-3775.

Drăguț, Lucian, Dirk Tiede, and Shaun R. Levick. 2010. "ESP: A Tool to Estimate Scale Parameter for Multiresolution Image Segmentation of Remotely Sensed Data." International Journal of Geographical Information Science 24 (6): 859871.

Duro, Dennis C., Steven E. Franklin, and Monique G. Dubé. 2012. “A Comparison of Pixel-Based and Object-Based Image Analysis with Selected Machine Learning Algorithms for the Classification of Agricultural Landscapes using SPOT-5 HRG Imagery." Remote Sensing of Environment 118: 259-272.

Espindola, G. M., G. Camara, I. A. Reis, L. S. Bins, and A. M. Monteiro. 2006. "Parameter Selection for Region-Growing Image Segmentation Algorithms using Spatial Autocorrelation." International Journal of Remote Sensing 27 (14): 3035-3040.

Eysn, Lothar, Markus Hollaus, Klemens Schadauer, and Norbert Pfeifer. 2012. "Forest Delineation Based on Airborne LIDAR Data." Remote Sensing 4: 762-783. doi:10.3390/rs4030762.

Falkowski, Michael J., Michael A. Wulder, Joanne C. White, and Mark G. Gillis. 2009. "Supporting Large-Area, Sample-Based Forest Inventories with Very High Spatial Resolution Satellite Imagery." Progress in Physical Geography 33 (3): 403-423.

Feitosa, R. Q., G. A. O. P. Costa, T. B. Cazes, and B. Feijo. 2006. "A Genetic Approach for the Automatic Adaptation of Segmentation Parameters." In Bridging Remote Sensing and GIS: 1st International Conference on Object-based Image Analysis (OBIA 2006), edited by: Stefan Lang, Thomas Blaschke, and Elisabeth Schöpfer. International Archives of the Photogrammetry, Remote Sensing and Spatial Information Sciences - Volume XXXVI/4-C42.

Freeman, T. Graham. 1991. "Calculating catchment area with divergent flow based on a regular grid." Computers and Geosciences 17: 413-422.Gao, Yan, Jean Francois Mas, Norman Kerle, and Jose Antonio Navarrete Pacheco. 2011. "Optimal Region Growing Segmentation and Its Effect on Classification Accuracy." International Journal of Remote Sensing 32 (13): 3747-3763.

Geerling, G. W., M. Labrador-Garcia, J. P. G. W. Clevers, A. M. J. Ragas, and A. J. M. Smits. 2007. "Classification of Floodplain Vegetation by Data-Fusion of 
Spectral (CASI) and LiDAR Data." International Journal of Remote Sensing 28 (19): 4263-4284.

Geerling, G. W., M. J. Vreeken-Buijs, P. Jesse, A. M. J. Ragas, and A. J. M. Smits. 2009. "Mapping River Floodplain Ecotopes by Segmentation of Spectral (CASI) and Structural (LiDAR) Remote Sensing Data." River Research and Applications 25: 795-813.

Gonzales, Rafael C., and Richard E. Woods. 2002. Digital Image Processing. 2nd ed. Upper Saddle River: Prentice Hall.

Grabs, T., Jan Seibert, K. Bishop, and H. Laudon. 2009. "Modeling Spatial Patterns of Saturated Areas: A Comparison of the Topographic Wetness Index and a Dynamic Distributed Model. Journal of Hydrology 373: 15-23.

Güntner, Andreas, Jan Seibert, and Stefan Uhlenbrook. 2004. "Modeling Spatial Patterns of Saturated Areas: An Evaluation of Different Terrain Indices." Water Resources Research 40, W05114. doi:10.1029/2003WR002864.

Hay, Geoffrey. J., Thomas Blaschke, Danielle J. Marceau, and André Bouchard. 2003. "A Comparison of Three Image-Object Methods for the Multiscale Analysis of Landscape Structure." ISPRS Journal of Photogrammetry and Remote Sensing 57: $327-345$.

Hay, Geoffrey J., Guillermo Castilla, Michael A. Wulder, and Jose R. Ruiz 2005. “An Automated Object-Based Approach for the Multiscale Image Segmentation of Forest Scenes. International Journal of Applied Earth Observation and Geoinformation 7: 339-359.

Hou, Zhengyang, Qing Xu, Tuula Nuutinen, and Timo Tokola. 2013. "Extraction of remote sensing-based forest management units in tropical forests". Remote Sensing of Environment 130: 1-10.

Isenburg, Martin. 2011. LAStools: Efficient Tools for LiDAR Processing. Version 110815. http://lastools.org.

Ji, Xiaole, Yanchen Bo, Jiehai Cheng, Yaqian He, and Xiaolong Liu. 2012. "The Method of Assessing Shape Similarity of Object-Based Classification Result of Remote Sensing Imagery". In Proceedings of the Second International Workshop on Earth Observation and Remote Sensing Applications (EORSA 2012), 157-160. IEEE. http://ieeexplore.ieee.org/xpl/mostRecentIssue.jsp?punumber=6248407/.

Ke, Yinghai, Lindi J. Quackenbush, and Jungho Im. 2010. "Synergistic Use of QuickBird Multispectral Imagery and LIDAR Data for Object-Based Forest Species Classification.” Remote Sensing of Environment 114: 1141-1154.

Kim, Minho, Marguerite Madden, and Timothy A. Warner. 2008. "Estimation of Optimal Image Object Size for the Segmentation of Forest Stands with Multispectral IKONOS Imagery." In Object-Based Image Analysis: Spatial Concepts for Knowledge-Driven Remote Sensing Applications, edited by Thomas Blaschke, Stefan Lang, and Geoffrey J. Hay, 291-307. Berlin: Springer.

Kim, Minho, Marguerite Madden, and Timothy A. Warner. 2009. "Forest Type Mapping using Object-specific Texture Measures from Multispectral Ikonos Imagery: Segmentation Quality and Image Classification Issues." Photogrammetric Engineering \& Remote Sensing 75 (7): 819-829.

Kim, Minho, Timothy A. Warner, Marguerite Madden, and Douglas S. Atkinson. 2011. "Multi-Scale GEOBIA with Very High Spatial Resolution Digital Aerial 
Imagery: Scale, Texture and Image Objects." International Journal of Remote Sensing 32 (10): 2825-2850

Kopecký, Martin and Stěpánka Č́žzová. 2010. "Using Topographic Wetness Index in Vegetation Ecology: Does the Algorithm Matter?" Applied Vegetation Science 13: 450-459.

Lang, Stefan, F. Albrecht, S. Kienberger, and Dirk Tiede. 2010. "Object Validity for Operational Tasks in a Policy Context.” Journal of Spatial Science 55 (1): 9-22.

Lawrence, Rick L., Shana D. Wood, and Roger L. Sheley. 2006. "Mapping Invasive Plants using Hyperspectral Imagery and Breiman Cutler Classifications (RandomForest).” Remote Sensing of Environment 100: 356-362.

Leckie, Donald G., François A. Gougeon, Nicholas Walsworth, and Dennis Paradine. 2003. "Stand Delineation and Composition Estimation using Semi-Automated Individual Tree Crown Analysis". Remote Sensing of Environment 85: 355-369.

Li, Haitao, Haiyan Gu, Yanshun Han, and Jinghui Yang. 2010. "Object-Oriented Classification of High-Resolution Remote Sensing Imagery based on an Improved Colour Structure Code and a Support Vector Machine." International Journal of Remote Sensing 31 (6): 1453-1470.

Li, Nan, Hong Huo, and Tao Fang. 2010. "A Novel Texture-Preceded Segmentation Algorithm for High-Resolution Imagery." IEEE Transactions on Geoscience and Remote Sensing 48 (7): 2818-2828.

Liaw, Andy, and Matthew Wiener. 2002. "Classification and Regression by randomForest." $R$ News 2/3: 18-22.

Lucieer, Arko, and Alfred Stein. 2002. "Existential Uncertainty of Spatial Objects Segmented From Satellite Sensor Imagery.” IEEE Transactions on Geoscience and Remote Sensing 40 (11): 2518-2521.

Marpu, P. R., M. Neubert, H. Herold, and I. Niemeyer. 2010. "Enhanced Evaluation of Image Segmentation Results". Journal of Spatial Science 55 (1): 55-68.

Meinel, G., and M. Neubert. 2004. "A Comparison of Segmentation Programs for High Resolution Remote Sensing Data.” In XX ISPRS Congress: Technical Commission IV, edited by Orhan Altan, 1097-1105. International Archives of the Photogrammetry, Remote Sensing and Spatial Information Sciences Volume XXXV Part B4.

Möller, M., L. Lymburner, and M. Volk. 2007. "The Comparison Index: A Tool for Assessing the Accuracy of Image Segmentation." International Journal of Applied Earth Observation and Geoinformation 9: 311-321.

Mustonen, Jukka, Petteri Packalén, and Annika Kangas. 2008. "Automatic Segmentation of Forest Stands Using a Canopy Height Model and Aerial Photography." Scandinavian Journal of Forest Research 23 (6): 534-545.

Murphy, P. N. C., J. Ogilvie, and P. Arp. 2009. "Topographic Modeling of Soil Moisture Conditions: a Comparison and Verification of Two Models." European Journal of Soil Science 60: 94-109.

Neubert, M., and H. Herold. 2008. "Assessment of Remote Sensing Image Segmentation Quality." In GEOBIA 2008 - Pixels, Objects, Intelligence: GEOgraphic Object Based Image Analysis for the 21st Century Proceedings, edited by Geoffrey J. Hay, Thomas Blaschke and Danielle Marceau. International Archives of Photogrammetry, Remote Sensing and Spatial Information Sciences XXXVIII-4/C1. 
Neubert, M., and G. Meinel. 2003. "Evaluation of Segmentation Programs for High Resolution Remote Sensing Applications." In Proceedings Joint ISPRS/EARSeL Workshop "High Resolution Mapping from Space 2003”, edited by M. Schroeder, K. Jacobsen, and C. Heipke. http://www.ipi.unihannover.de/fileadmin/institut/pdf/neubert.pdf.

Neubert, M., H. Herold, and G. Meinel. 2008. “Assessing Image Segmentation Quality - Concepts, Methods and Applications." In Object-Based Image Analysis: Spatial Concepts for Knowledge-Driven Remote Sensing Applications, edited by Thomas Blaschke, Stefan Lang, and Geoffrey J. Hay, 769-784. Berlin: Springer. Pekkarinen, Anssi. 2002. "Image Segment-Based Spectral Features in the Estimation of Timber Volume." Remote sensing of Environment 82: 349-359.

R Development Core Team. 2012. R: A Language and Environment for Statistical Computing. Vienna: R Foundation for Statistical Computing. ISBN 3-90005107-0, URL http://www.R-project.org/.

Radoux, J. and P. Defourny. 2007. "A Quantitative Assessment of Boundaries in Automated Forest Stand Delineation using Very High Resolution Imagery." Remote Sensing of Environment 110: 468-475.

Rodriguez-Galiano, V. F., B. Ghimire, J. Rogan, M. Chica-Olmo, and J. P. RigolSanchez. 2012. "An Assessment of the Effectiveness of a Random Forest Classifier for Land-Cover Classification." ISPRS Journal of Photogrammetry and Remote Sensing 67: 93-104.

Smith, A. 2010. "Image Segmentation Scale Parameter Optimization and Land Cover Classification using the Random Forest Algorithm." Journal of Spatial Science 55(1): 69-79.

Sørensen, R., U. Zinko, and Jan Seibert. 2006. "On the Calculation of the Topographic Wetness Index: an Evaluation of Different Methods based on Field Observations." Hydrology and Earth System Sciences 10: 101-112.

Tian, J. and D.-M. Chen. 2007. "Optimization in Multi-Scale Segmentation of HighResolution Satellite Images for Artificial Feature Recognition.” International Journal of Remote Sensing 28 (20): 4625-4644.

Verbeeck, Klaartje, Martin Hermy, and Jos van Orshoven. 2012. "External GeoInformation in the Segmentation of VHR imagery Improves the Detection of Imperviousness in Urban Neighborhoods." International Journal of Applied Earth Observation and Geoinformation 18: 428-435.

Vesterbacka, Raisa. 2010. Luontopalvelujen luontotyyppi-inventoinnin maastotyöohje. Vantaa: Metsähallitus.

Wang, L., W. Sousa, and Peng Gong. 2004. "Integration of Object-Based and PixelBased Classification for Mapping Mangroves with IKONOS Imagery." International Journal of Remote Sensing 25 (24): 5655-5668.

Wang, Zhongwu, John R. Jensen, and Jungho Im. 2010. "An Automatic Region-Based Image Segmentation Algorithm for Remote Sensing Applications." Environmental Modelling and Software 25: 1149-1165.

Weidner, Uwe 2008. "Contribution to the Assessment of Segmentation Quality for Remote Sensing Applications." In XXIst ISPRS Congress: Technical Commission VII, edited by Chen Jun, Jiang Jie, and John van Genderen, 479484. International Archives of Photogrammetry, Remote Sensing and Spatial Information Sciences - Volume XXXVII Part B7. 
967 Whiteside, Timothy G., Guy S. Boggs, and Stefan W. Maier. 2011. "Comparing Object$968 \quad$ Based and Pixel-Based Classifications for Mapping Savannas." International 969 Journal of Applied Earth Observation and Geoinformation 13: 884-893.

970 Wulder, Michael A., Joanne C. White, Geoffrey J. Hay, and Guillermo Castilla. 2008.

971 "Towards Automated Segmentation of Forest Inventory Polygons on High

972

973

974

975

976 Spatial Resolution Satellite Imagery." The Forestry Chronicle 84 (2): 221-230.

Yang, Luren, Fritz Albregtsen, Tor Lønnestad, and Per Grøttum. 1995. "A Supervised Approach to the Evaluation of Image Segmentation Methods." Computer Analysis of Images and Patterns: 6thInternational Conference, CAIP '95 Prague Czech Republic, September 6-8, 1995 Proceedings, edited by Václac Hlaváč, and Radim Šára, 759-765. Lecture Notes on Computer Science, Volume 970. Heildelberg: Springer.

Yue, Anzhi, Jianyu Yang, Chao Zhang, Wei Su, Wenju Yun, Dehai Zhu, Shunxi Liu, and Zhongwu Wang. 2012. "The Optimal Segmentation Scale Identification Using Multispectral WorldView-2 Images”. Sensor Letters 10 (1-2): 285291(7).

Zhan, Qingming, Martien Molenaar, Klaus Tempfli, and Wengzhong Shi. 2005. "Quality Assessment for Geo-Spatial Objects Derived from Remotely Sensed Data." International Journal of Remote Sensing 26 (14): 2593-2974.

Zhang, Hui, Jason E. Fritts, and Sally A. Goldman. 2008. "Image Segmentation Rvaluation: A Survey of Unsupervised Methods." Computer Vision and Image Understanding 110: 260-280.

Zhang, Y. J. 1996. "A Survey on Evaluation Methods for Image Segmentation.” Pattern Recognition 29 (8): 1335-1346.

Zhang, Y. J. 1997. "Evaluation and Comparison of Different Segmentation Algorithms.” Pattern Recognition Letters 18 (10): 963-974. 
993 Table 1. Different habitat types that were mapped during field work when the reference 994 polygons were drawn and that were used in the classification part of the research.

\begin{tabular}{ll}
\hline Habitat type & Number of age groups/management possibilities \\
\hline xeric (pine dominated) forests & 4: clear-cut, sapling stand, young, mature \\
mesic (spruce dominated) forests & 5: clear-cut, sapling stand, young, mature, natural \\
herb-rich (mixed/deciduous) forests & 4 : sapling stand, young, mature, natural \\
bare rock & 1 \\
pine mires & 1 \\
spruce mires & 2 : not drained, drained \\
open mires & 1 \\
water (lakes and streams) & 1 \\
small creeks & 1 \\
springs & 1 \\
grasslands & 1 \\
fields & 1 \\
roads & 1 \\
yards & 1 \\
sand pits & 1 \\
\hline
\end{tabular}

995

996 Table 2. Simple segmentation goodness evaluation measures that were used in 997 segmentation evaluation. Column MEASURES refers to what the method should 998 measure. Column SOURCE refers to the article where the measure was first used. 999 Column WEIGHTED refers to if the measure is weighted by a reference object.

\begin{tabular}{|c|c|c|c|c|}
\hline METHOD & MEASURES & SOURCE & WEIGHTED & NOTE \\
\hline UnderMerging & undersegmentation & Yang et al. (1995) & & \\
\hline OverMerging & oversegmentation & Yang et al. (1995) & & \\
\hline AFI & area match & Lucieer \& Stein (2002) & & \\
\hline CountOver & oversegmentation & Lucieer \& Stein (2002) & & based on AFI \\
\hline CountUnder & undersegmentation & Lucieer \& Stein (2002) & & based on AFI \\
\hline SimSize mean & area match & Zhan et al. (2005) & $\mathrm{X}$ & \\
\hline SimSize sd & area match & Zhan et al. (2005) & $\mathrm{X}$ & \\
\hline RAsuper & undersegmentation & Möller, Lymburner, and Volk (2007) & $\mathrm{X}$ & \\
\hline RAsub & oversegmentation & Möller, Lymburner, and Volk (2007) & $\mathrm{X}$ & \\
\hline QR & area match & Weidner (2008) & $\mathrm{X}$ & \\
\hline OverSegmentation & oversegmentation & Clinton et al. (2010) & $\mathrm{X}$ & \\
\hline UnderSegmentation & undersegmentation & Clinton et al. (2010) & $\mathrm{X}$ & \\
\hline RPsuper & distance to centroid & Möller et al. (2007) & $\mathrm{X}$ & \\
\hline RPsub & distance to centroid & Möller et al. (2007) & $X$ & \\
\hline QLoc mean & distance to centroid & Zhan et al. (2005) & $\mathrm{X}$ & $=\mathrm{RPsub}$ \\
\hline QLoc sd & distance to centroid & Zhan et al. (2005) & $\mathrm{X}$ & \\
\hline
\end{tabular}


1002 Table 3. Combined segmentation goodness evaluation measures that were used in 1003 segmentation evaluation. Column INCLUDES refers to the simple measures that are 1004 included in the respective combined measure. Column CALCULATION refers to how 1005 the combined measure was calculated.

\begin{tabular}{ccc}
\hline METHOD & INCLUDES & CALCULATION \\
\hline M & RAsuper, RAsub, RPsuper, RPsub & RMS \\
ZH1 & SimSize mean, SimSize std, QLoc mean, QLoc std & RMS \\
ZH2 & SimSize mean, QLoc mean & RMS \\
D & OverSegmentation, UnderSegmentation & RMS \\
OverUnder & CountOver, CountUnder & SUM \\
MergeSum & OverMerging, UnderMerging & SUM \\
COMBINED & all other simple measures than QLoc mean & RMS, normalized \\
\hline
\end{tabular}


1007 Table 4. Best segmentations according to different measures and reference polygons. Segmentation methods are marked as follows. Text 1008 refers to method, letter after the text to layer combination a-d (see text), s to scale (FNEA) or similarity parameter (IDRISI), $\mathrm{c}$ to colour 1009 parameter, $\mathrm{m}$ to weight given to mean and $\mathrm{v}$ to weight given to variance.

\begin{tabular}{|c|c|c|c|c|c|c|c|c|}
\hline MEASURE & All area & SA1 & SA2 & SA3 & water & mires & FP & FFPS \\
\hline UnderMerging & FNEA_d_s5_c.75 & FNEA_d_s5_c.75 & FNEA_a_s5_c. 75 & FNEA_a_s5_c.75 & FNEA_a_s5_c. 5 & FNEA_d_s5_c.75 & FNEA_c_s5_c.75 & FNEA_a_s5_c.5 \\
\hline OverMerging & FNEA_b_s50_c.25 & FNEA_b_s50_c.25 & FNEA_b_s50_c.5 & FNEA_b_s50_c.5 & IDRISI_b_s70_m1_v9 & FNEA_b_s50_c.25 & FNEA_b_s50_c.25 & FNEA_c_s50_c.75 \\
\hline AFI & IDRISI_a_s25_m9_v1 & IDRISI_a_s25_m1_v9 & IDRISI_d_s20_m9_v1 & IDRISI_c_s35_m1_v9 & IDRISI_d_s70_m5_v5 & FNEA_b_s5_c.25 & IDRISI_a_S45_m5_v5 & IDRISI_b_s35_m9_v1 \\
\hline CountOver & FNEA_b_s50_c.25 & FNEA_b_s50_c. 25 & FNEA_b_s50_c.25 & FNEA_c_s50_c. $25^{*}$ & FNEA_b_s50_c.5 & FNEA_d_s45_c. $25^{*}$ & FNEA_b_s50_c. $5^{*}$ & FNEA_b_s50_c. $75^{*}$ \\
\hline CountUnder & IDRISI_a_s20_m1_v9* & IDRISI_a_s25_m1_v9* & IDRISI_a_s20_m9_v1* & IDRISI_b_550_m9_v1 & FNEA_b_s25_c. $25^{*}$ & IDRISI_a_s40_m1_v9* & IDRISI_a_s70_m1_v9* & IDRISI_a_s70_m1_v9* \\
\hline SimSize mean & FNEA_b_s30_c.75 & FNEA_b_s30_c.75 & FNEA_d_s30_c.75 & IDRISI_c_s65_m9_v1 & FNEA_b_s45_c.75 & IDRISI_c_s55_m9_v1 & FNEA_c_s30_c.25 & FNEA_d_s40_c.25 \\
\hline SimSize sd & FNEA_a_s5_c.75 & FNEA_b_s5_c.75 & FNEA_a_s5_c.75 & FNEA_a_s5_c.75 & FNEA_a_s5_c.5 & FNEA_b_s50_c. 25 & FNEA_a_s5_c.75 & FNEA_d_s5_c.75 \\
\hline RAsuper & FNEA_b_s5_c.75 & FNEA_b_s5_c.75 & FNEA_b_s5_c.75 & FNEA_a_s5_c.75 & FNEA_c_s5_c.5 & FNEA_d_s5_c.75 & FNEA_b_s5_c.75 & FNEA_b_s5_c.75 \\
\hline RAsub & FNEA_b_s50_c.25 & FNEA_b_s50_c.25 & FNEA_b_s50_c.5 & FNEA_b_s50_c.25 & FNEA_b_s50_c.5 & FNEA_b_s50_c.25 & FNEA_b_s50_c.25 & FNEA_b_s50_c.25 \\
\hline QR & FNEA_b_s35_c.75 & FNEA_b_s30_c.75 & FNEA_c_s30_c.75 & FNEA_a_s45_c. 75 & IDRISI_b_s65_m9_v1 & IDRISI_a_s45_m1_v9 & FNEA_c_s45_c. 75 & FNEA_d_s40_c.75 \\
\hline OverSegmentation & FNEA_b_s50_c.5 & FNEA_b_s50_c. 25 & FNEA_b_s50_c.5 & FNEA_b_s50_c.5 & IDRISI_b_s70_m1_v9 & FNEA_b_s50_c.25 & FNEA_b_s50_c. 25 & FNEA_c_s50_c.75 \\
\hline UnderSegmentation & FNEA_b_s5_c.75 & FNEA_b_s5_c.75 & FNEA_b_s5_c.75 & FNEA_b_s5_c.75 & IDRISI_a_s20_m5_v5 & FNEA_b_s5_c.75 & FNEA_b_s5_c.75 & FNEA_b_s5_c.75 \\
\hline RPsuper & IDRISI_d_555_m9_v1 & FNEA_b_s20_c.5 & FNEA_d_s20_c. 5 & FNEA_b_s15_c.5 & IDRISI_b_s65_m9_v1 & FNEA_d_s10_c.25 & FNEA_d_s30_c.75 & IDRISI_d_s60_m9_v1 \\
\hline RPsub & FNEA_b_s35_c.75 & IDRISI_b_s55_m5_v5 & FNEA_a_s40_c. 75 & FNEA_c_s40_c.75 & IDRISI_b_s65_m9_v1 & FNEA_b_s15_c. 25 & FNEA_c_s30_c.75 & FNEA_d_s40_c.75 \\
\hline QLoc mean & FNEA_b_s35_c.75 & IDRISI_b_555_m5_v5 & FNEA_a_s40_c.75 & FNEA_c_s40_c.75 & IDRISI_b_s65_m9_v1 & FNEA_b_s15_c.25 & FNEA_c_s30_c.75 & FNEA_d_s40_c.75 \\
\hline QLoc sd & FNEA_a_s45_c. 25 & IDRISI_b_s65_m9_v1 & FNEA_c_s45_c. 75 & FNEA_c_s40_c.75 & IDRISI_b_s65_m1_v9 & FNEA_b_s20_c.25 & FNEA_c_s30_c.75 & FNEA_c_s50_c.25 \\
\hline M & FNEA_c_s25_c.75 & IDRISI_b_s45_m9_v1 & FNEA_a_s5_c.5 & FNEA_b_s20_c.75 & FNEA_c_s45_c.75 & FNEA_d_s5_c.75 & FNEA_b_s5_c.75 & FNEA_a_s25_c.5 \\
\hline ZH1 & FNEA_d_s50_c.25 & FNEA_d_s50_c.25 & FNEA_c_s5_c.25 & FNEA_b_s45_c. 5 & IDRISI_b_s40_m5_v5 & FNEA_b_s50_c. 25 & FNEA_a_s5_c.25 & FNEA_a_s45_c. 25 \\
\hline OverUnder & IDRISI_b_s70_m9_v1 & IDRISI_b_s65_m1_v9 & IDRISI_a_s70_m5_v5 & IDRISI_b_s70_m9_v1 & FNEA_b_s50_c.5 & IDRISI_a_s70_m9_v1 & FNEA_c_s50_c.75 & IDRISI_a_s70_m1_v9 \\
\hline MergeSum & FNEA_b_s15_c.5 & FNEA_b_s15_c. 5 & FNEA_a_s15_c.75 & FNEA_b_s20_c.75 & IDRISI_b_s65_m9_v1 & FNEA_b_s10_c. 25 & FNEA_c_s25_c. 5 & FNEA_d_s25_c.25 \\
\hline COMBINED & FNEA_b_s25_c. 5 & IDRISI_b_s50_m5_v5 & FNEA_b_s45_c.75 & FNEA_b_s30_c.75 & IDRISI_b_s70_m1_v9 & FNEA_a_s40_c. 5 & FNEA_b_s45_c. 5 & FNEA_a_s35_c.25 \\
\hline
\end{tabular}

*=tie with other segmentations which are not indicated here. Segmentation that is shown ranked best using OverUnder evaluation method

1010

1011 
1012

1013 Table 5. 20 best and 10 worst segmentations based on all area reference polygons and 1014 COMBINED measure. Segmentation methods are named as in Table 4.

\begin{tabular}{llc}
\hline & SEGMENTATION & COMBINED \\
\hline 1 & FNEA_b_s25_c.5 & 0.646 \\
2 & FNEA_b_s25_c.75 & 0.649 \\
3 & FNEA_b_s30_c.75 & 0.650 \\
4 & FNEA_c_s25_c.75 & 0.650 \\
5 & FNEA_d_s25_c.75 & 0.651 \\
6 & IDRISI_b_s55_m9_v1 & 0.652 \\
7 & FNEA_a_s30_c.75 & 0.652 \\
8 & IDRISI_b_s65_m9_v1 & 0.652 \\
9 & IDRISI_d_s65_m9_v1 & 0.652 \\
10 & FNEA_d_s30_c.75 & 0.652 \\
11 & IDRISI_b_s50_m5_v5 & 0.653 \\
12 & FNEA_b_s20_c.5 & 0.653 \\
13 & IDRISI_b_s55_m5_v5 & 0.653 \\
14 & IDRISI_b_s60_m9_v1 & 0.653 \\
15 & IDRISI_b_s50_m9_v1 & 0.653 \\
16 & IDRISI_c_s60_m5_v5 & 0.653 \\
17 & IDRISI_a_s60_m1_v9 & 0.653 \\
18 & IDRISI_a_s70_m9_v1 & 0.653 \\
19 & IDRISI_d_s65_m5_v5 & 0.654 \\
20 & IDRISI_a_s60_m5_v5 & 0.654 \\
$\ldots$ & & \\
243 & IDRISI_c_s20_m9_v1 & 0.770 \\
244 & FNEA_a_s5_c.25 & 0.771 \\
245 & FNEA_ccs5_c.5 & 0.782 \\
246 & FNEA_d_s5_c.5 & 0.789 \\
247 & FNEA_a_s5_c.5 & 0.796 \\
248 & FNEA_b_s5_c.5 & 0.797 \\
249 & FNEA_c_s5_c.75 & 0.801 \\
250 & FNEA_d_s5_c.75 & 0.805 \\
251 & FNEA_a_s5_c.75 & 0.811 \\
252 & FNEA_b_s5_c.75 & 0.813 \\
\hline & &
\end{tabular}

1015

1016 
1017 Table 6. Best segmentations based on different measures sorted by segmentation 1018 method, layer combinations, and different parameter options. Numbers refer to the 1019 number of segmentations that were regarded as best.

\begin{tabular}{|c|c|c|c|c|c|c|c|c|}
\hline \multicolumn{3}{|c|}{ all segmentations } & \multicolumn{3}{|c|}{ FNEA segmentations } & \multicolumn{3}{|c|}{ IDRISI segmentations } \\
\hline & value & count & & value & count & & value & count \\
\hline \multirow[t]{3}{*}{ method } & FNEA & 140 & scale & 5 & 36 & similarity & 20 & 4 \\
\hline & IDRISI & 44 & & 10 & 2 & & 25 & 3 \\
\hline & & & & 15 & 6 & & 30 & 0 \\
\hline \multirow[t]{12}{*}{ layers } & $\mathrm{a}$ & 35 & & 20 & 6 & & 35 & 2 \\
\hline & $\mathrm{b}$ & 93 & & 25 & 6 & & 40 & 2 \\
\hline & $\mathrm{c}$ & 28 & & 30 & 13 & & 45 & 3 \\
\hline & d & 28 & & 35 & 10 & & 50 & 2 \\
\hline & & & & 40 & 12 & & 55 & 5 \\
\hline & & & & 45 & 13 & & 60 & 2 \\
\hline & & & & 50 & 36 & & 65 & 9 \\
\hline & & & & & & & 70 & 12 \\
\hline & & & colour & 0.25 & 40 & & & \\
\hline & & & & 0.5 & 30 & mean/var & $0.1 / 0.9$ & 15 \\
\hline & & & & 0.75 & 70 & & $0.5 / 0.5$ & 8 \\
\hline & & & & & & & $0.9 / 0.1$ & 21 \\
\hline
\end{tabular}

1020

1021 Table 7. Correlations between the COMBINED goodness measure and individual 1022 goodness measures based on different reference polygons. SA refers to sub-area, FP to 1023 forestry planning data and FFPS for FFPS data.

\begin{tabular}{lcccccccc}
\hline MEASURE & ALL & SA1 & SA2 & SA3 & water & mires & FP & FFPS \\
\hline UnderMerging & -0.24 & -0.39 & -0.63 & -0.13 & -0.63 & -0.61 & -0.69 & -0.33 \\
OverMerging & 0.89 & 0.90 & 0.95 & 0.90 & 0.88 & 0.97 & 0.91 & 0.90 \\
AFI & -0.22 & -0.36 & -0.63 & -0.10 & -0.40 & -0.61 & -0.54 & -0.24 \\
CountOver & 0.69 & 0.78 & 0.91 & 0.73 & 0.87 & 0.91 & 0.88 & 0.71 \\
CountUnder & -0.25 & -0.31 & -0.73 & -0.31 & -0.53 & -0.62 & -0.53 & -0.10 \\
SimSize mean & 0.89 & 0.91 & 0.91 & 0.89 & 0.85 & 0.29 & 0.92 & 0.82 \\
SimSize sd & -0.91 & -0.93 & -0.93 & -0.91 & -0.94 & -0.34 & -0.93 & -0.85 \\
RAsuper & -0.79 & -0.86 & -0.94 & -0.83 & -0.95 & -0.93 & -0.96 & -0.86 \\
RAsub & 0.43 & 0.54 & 0.80 & 0.53 & 0.86 & 0.75 & 0.85 & 0.52 \\
QR & 0.91 & 0.94 & 0.93 & 0.92 & 0.95 & 0.35 & 0.95 & 0.90 \\
OverSegmentation & 0.48 & 0.60 & 0.79 & 0.56 & 0.81 & 0.84 & 0.84 & 0.50 \\
UnderSegmentation & -0.52 & -0.65 & -0.82 & -0.58 & -0.79 & -0.86 & -0.87 & -0.63 \\
RPsuper & 0.85 & 0.67 & 0.52 & 0.78 & 0.88 & -0.55 & 0.74 & 0.96 \\
RPsub & 0.86 & 0.94 & 0.88 & 0.94 & 0.95 & 0.01 & 0.92 & 0.87 \\
QLoc mean & 0.86 & 0.94 & 0.88 & 0.94 & 0.95 & 0.01 & 0.92 & 0.87 \\
QLoc sd & 0.63 & 0.68 & 0.80 & 0.79 & 0.80 & 0.30 & 0.80 & 0.72 \\
M & 0.98 & 0.98 & -0.88 & 0.97 & -0.50 & -0.72 & -0.90 & 0.97 \\
ZH1 & 0.66 & 0.60 & -0.83 & 0.84 & 0.76 & -0.29 & -0.87 & 0.83 \\
ZH2 & 0.89 & 0.96 & 0.92 & 0.94 & 0.94 & 0.29 & 0.92 & 0.89 \\
D & 0.77 & 0.82 & 0.88 & 0.81 & 0.83 & 0.36 & 0.90 & 0.65 \\
OverUnder & 0.76 & 0.84 & 0.90 & 0.78 & 0.91 & 0.86 & 0.89 & 0.75 \\
MergeSum & 0.41 & 0.50 & -0.34 & 0.69 & 0.86 & -0.52 & 0.85 & 0.90 \\
\hline
\end{tabular}


1025 Table 8. Correlations between the COMBINED measure values based on different 1026 reference polygons.

\begin{tabular}{lcccccccc}
\hline & ALL & SA1 & SA2 & SA3 & water & mires & FP & FFPS \\
\hline ALL & 1.00 & 0.89 & 0.85 & 0.94 & 0.64 & 0.84 & 0.78 & 0.94 \\
SA1 & 0.89 & 1.00 & 0.83 & 0.84 & 0.59 & 0.83 & 0.79 & 0.88 \\
SA2 & 0.85 & 0.83 & 1.00 & 0.87 & 0.82 & 0.97 & 0.97 & 0.89 \\
SA3 & 0.94 & 0.84 & 0.87 & 1.00 & 0.73 & 0.87 & 0.83 & 0.88 \\
water & 0.64 & 0.59 & 0.82 & 0.73 & 1.00 & 0.80 & 0.80 & 0.63 \\
mires & 0.84 & 0.83 & 0.97 & 0.87 & 0.80 & 1.00 & 0.95 & 0.85 \\
FP & 0.78 & 0.79 & 0.97 & 0.83 & 0.80 & 0.95 & 1.00 & 0.84 \\
FFPS & 0.94 & 0.88 & 0.89 & 0.88 & 0.63 & 0.85 & 0.84 & 1.00 \\
\hline
\end{tabular}

1027 Table 9. Classification accuracies derived from classifications based on different 1028 segmentations. Segmentations are marked as in Table 2. Also, criteria why each 1029 segmentation was chosen to the classification analyses are given.

\begin{tabular}{lll}
\hline SEGMENTATION & ACC & Why segmentation was selected to classification \\
\hline FNEA_b_s5_c.75 & 0.69 & BEST in avoiding undersegmentation, WORST based on COMBINATION and ALL AREA \\
FNEA_c_s5_c.75 & 0.69 & Comparison against FNEA_b_s5_c.75 \\
FNEA_a_s10_c.5 & 0.71 & WV-2 layers only, comparison against FNEA_c_s10_c.5 \\
FNEA_c_s10_c.5 & 0.72 & BEST segmentation based on visual interpretation \\
FNEA_d_s15_c.25 & 0.71 & GOOD in visual interpretation \\
FNEA_a_s20_c.75 & 0.69 & Segmentation based on WV-2 data only, OK visually \\
FNEA_b_s25_c.5 & 0.66 & BEST segmentation based on COMBINATION and ALL AREA, OK visually \\
FNEA_d_s35_c.5 & 0.66 & BEST based on D and ALL AREA \\
FNEA_c_s50_c.75 & 0.65 & GOOD in avoiding oversegmentation, GOOD in visual boundary evaluation \\
IDRISI_d_s30_m9v1 & 0.70 & OK in visual interpretation, small segments \\
IDRISI_c_s40_m5v5 & 0.69 & OK in visual interpretation, quite small segments \\
IDRISI_b_s70_m9v1 & 0.60 & BEST based on OverUnder and ALL AREA, BAD in visual interpretation \\
\hline
\end{tabular}




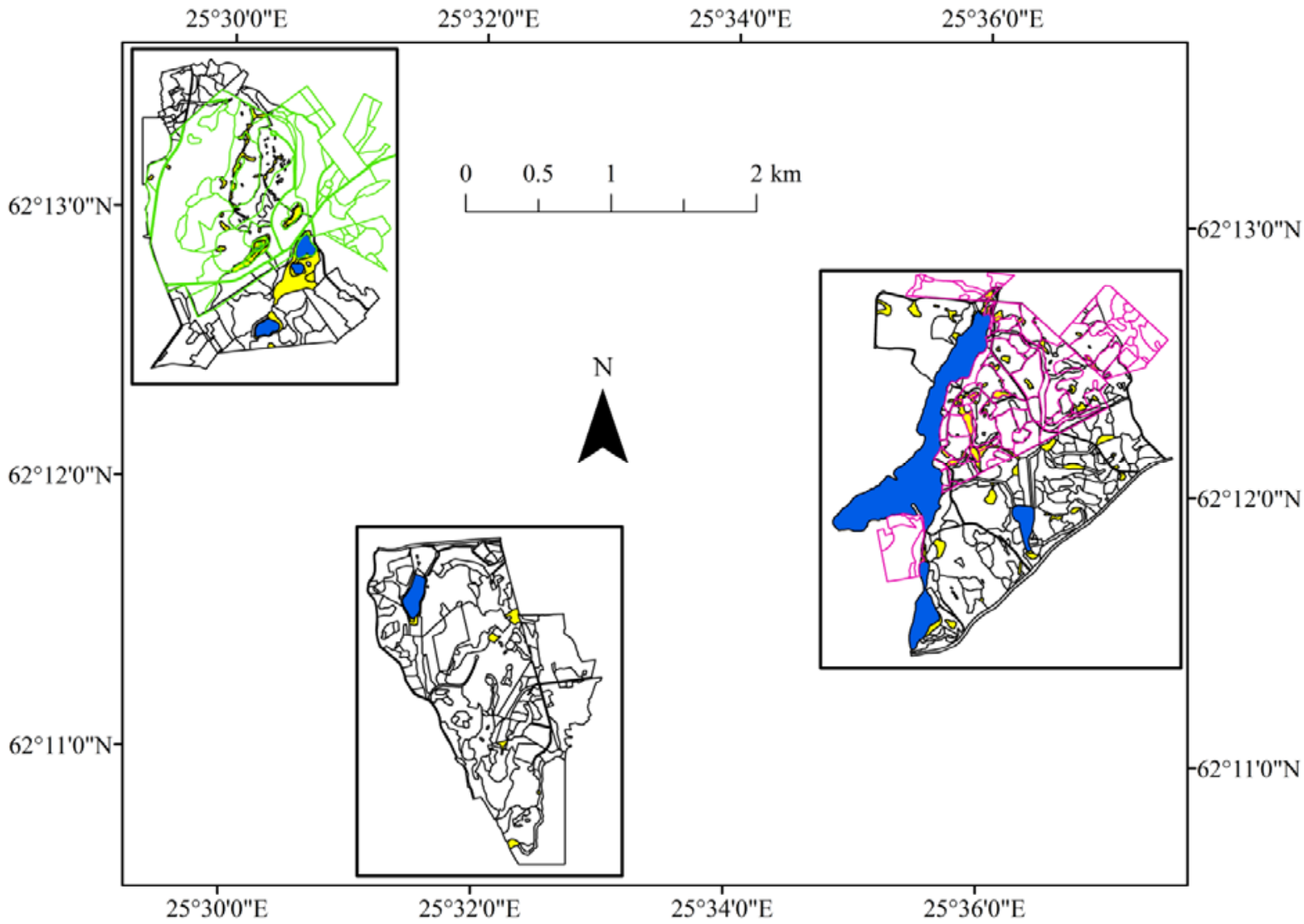

1032 Figure 1. Different reference polygons used. Reference polygons drawn in our field 1033 work are marked with black borders. Sub-area 1 is located in the eastern, sub-area 2 in 1034 the north-western and sub-area 3 in the southern part of the whole area. Water bodies 1035 are drawn with blue colour and mires with yellow colour. Forestry planning polygons 1036 1037 are marked with magenta outlines and FFPS polygons with green outlines. FNEA segmentations were performed inside the black rectangles whereas IDRISI segmentations were performed also in the areas between the black rectangles. 

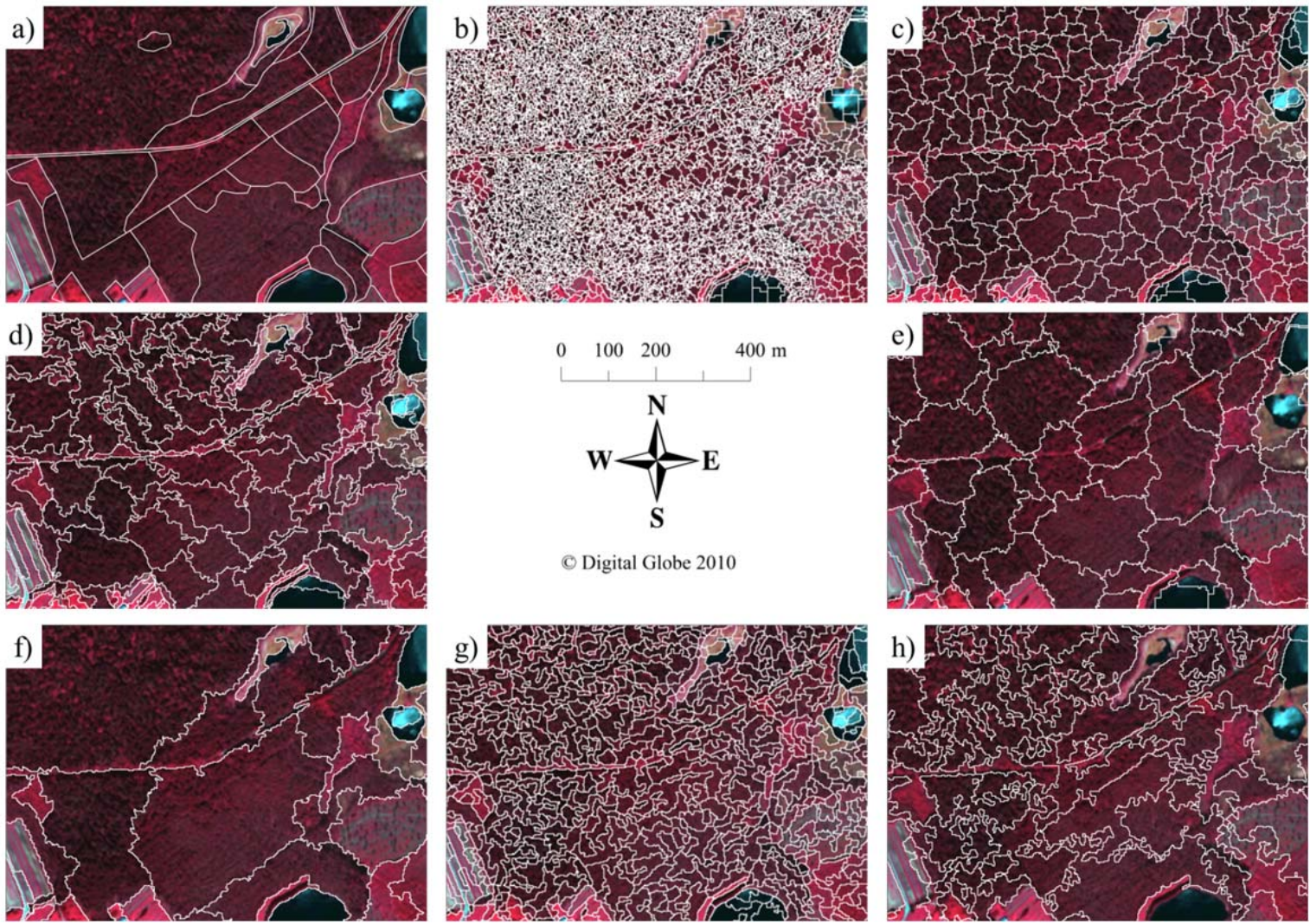

1040 Figure 2. Reference polygons and a visually chosen set of different segmentations

1041 drawn on a WV-2 false colour image (red: band 7/NIR1, green: band 5/red, blue: band

1042 3/green) in background. a) reference polygons, b) FNEA_b_s5_c.75, c)

1043 FNEA_c_s10_c.5, d) FNEA_a_s20_c.75, e) FNEA_b_s25_c.5, f) FNEA_c_s50_c.75,

1044 g) IDRISI_d_s30_m9v1, and h) IDRISI_b_s70_m9v1. Images are from the southern

1045 part of the sub-area 2. 